
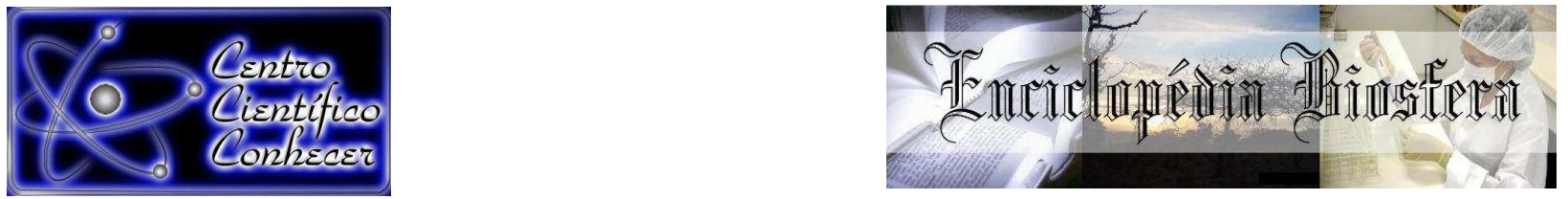

\title{
ESTUDO COMPARATIVO DE MODELOS CLÁSSICOS E TÉCNICAS DE COMBINAÇÃO PARA A PREVISÃO DO VOLUME DE CAPTAÇÃO DE ÁGUA PARA USO URBANO - UM ESTUDO DE CASO
}

Raphael Henrique Soares Machado ${ }^{1}$, José Donizetti de Lima², Dayse Regina Batistus $^{3}$, Gilson Oliveira Adamczuk ${ }^{4}$, Marcelo Gonçalves Trentin ${ }^{5}$

1 Acadêmico do Curso de Engenharia Elétrica e bolsista do PIBIC/Fundação Araucária-UTFPR - Câmpus Pato Branco

2 Professor do Programa de Pós-Graduação em Engenharia de Produção e Sistemas e do Departamento Acadêmico de Matemática da Universidade

Tecnológica Federal do Paraná, câmpus Pato Branco - donizetti@utfpr.edu.br 3 Professora do Departamento de Matemática da Universidade Tecnológica Federal do Paraná, câmpus Pato Branco

4 Professor do Programa de Pós-Graduação em Engenharia de Produção e Sistemas e do Departamento Acadêmico de Engenharia Mecânica da Universidade Tecnológica Federal do Paraná, câmpus Pato Branco

5 Professor do Programa de Pós-Graduação em Engenharia de Produção e Sistemas e do Departamento Acadêmico de Engenharia Elétrica da Universidade Tecnológica Federal do Paraná, câmpus Pato Branco

Recebido em: 08/04/2016 - Aprovado em: 30/05/2016 - Publicado em: 20/06/2016 DOI: 10.18677/Enciclopedia_Biosfera_2016_154

\begin{abstract}
RESUMO
O objetivo desse estudo foi investigar o desempenho de modelos clássicos e técnicas de combinação de séries temporais para modelar e prever o volume de água captado por uma empresa de saneamento básico no Sul do Brasil. A série disponível para análise continha uma base de 11 anos (2000 a 2011), com dados registrados mensalmente, sendo o ano de 2011 reservado para o processo de validação. Para atingir esse objetivo foram transcorridas algumas etapas: (i) caracterização da série temporal, a qual confirmou tendência e sazonalidade por meio de testes não paramétricos; (ii) ajuste e comparação dos modelos de suavização exponencial aditivo e multiplicativo de Holt-Winters (HW), com destaque para o modelo multiplicativo; (iii) ajuste e comparação dos modelos SARIMA com e sem aproximação, sendo que esse último modelo foi o que se revelou mais acurado; e (iv) aplicação de seis tipos de combinações dos melhores modelos HW e SARIMA, as quais apresentaram resultados mais acurados que os preditores individuais. Contudo, vale ressaltar que todos os modelos avaliados apresentaram resultados promissores, pois os MAPEs foram inferiores a 3\%.
\end{abstract}

PALAVRAS-CHAVE: Demanda de água de uso urbano; séries temporais; técnicas de combinação de previsão. 


\title{
A COMPARATIVE STUDY OF CLASSICAL TIME SERIES MODELS AND THEIR COMBINATION FOR PREDICTION OF ADDUCTED WATER FOR URBAN UTILIZATION
}

\begin{abstract}
This study investigated the performance of classic models and techniques for time series forecasting and their combination. Combination approach was used to model and predict the water volume adducted in water and sanitation company south of Brazil. The available twelve-year historical data set (2000-2011), monthly recorded, was divided into eleven-year adjustment stage (131-month data) and one-year validation stage (12-month data). The combination strategy comprised: (i) time series characterization, which We observed trend and seasonal patterns through nonparametric tests; (ii) adjustment and comparison of additive exponential smoothing model and multiplicative Holt-Winters model (HW); (iii) adjustment and comparison of SARIMA models; and (iv) six HW-SARIMA combination which presented the better results than the pure models (MAPE $=2,16 \%$ ). However, all results (pure models and models combination) were promising (MAPEs $<3 \%$ ).
\end{abstract}

KEYWORDS: urban water demand, time series, forecasts combination techniques.

\section{INTRODUÇÃO}

A água é um recurso natural, pivô sobre o qual as atividades sociais e econômicas e funções do ecossistema dependem. A gestão da água requer mecanismos governamentais que realizem considerações sobre a mesma, saindo da marginalidade administrativa governamental para o centro da sociedade. Seja em escala nacional ou local, por financiamento de estruturas e/ou adequação das existentes com novos financiamentos, esses mecanismos devem propiciar proteção aos recursos hídricos, garantindo o desenvolvimento sustentável com distribuição igualitária dos benefícios derivados da água (WWAP, 2012).

Segundo o relatório "Our Commom Future", elaborado pela Comissão de Brundtland, desenvolvimento sustentável é uma estrutura integrada de políticas ambientais e estratégias de desenvolvimento sociais e econômicos no sentido mais amplo. Contudo, muitas vezes o termo "desenvolvimento" é empregado somente com referência aos processos de mudanças econômicas. A conotação ampla procura atender as necessidades e aspirações do presente, sem comprometer a possibilidade de atendê-las no futuro (CMMAD, 1991). Este conceito que é ainda atual no contexto dos recursos hídricos, identificado no relatório "World Urbanization Prospects", elaborado pela Organização das Nações Unidas (ONU), projeta um crescimento populacional mundial em torno de $66 \%$, nos centros urbanos. Isso gera incertezas sobre a quantidade demandada de água para a produção de alimentos, energia, entre outros usos humanos e consequentemente, sobre a sustentabilidade do ecossistema (WUP, 2014).

O Brasil não se encontra distante das projeções mundiais de crescimento populacional. Considerado um país de dimensões continentais, composto por 27 unidades federativas, apresenta uma previsão conforme o relatório "World Population Prospects", revisão de 2015, de um crescimento populacional do atual 207,8 milhões para 238,2 milhões de brasileiros em 2050 (WPP, 2015). O país, dotado de uma ampla variedade climática e distintos ecossistemas tem, desde as regiões semiáridas à Amazônia, um elevado potencial de recursos hídricos. Assim, nos principais aglomerados urbanos do país, caracterizados por crescente adensamento demográfico, dinamismo econômico e importância estratégica, 
concentra-se os desafios de ordem técnica para o abastecimento de água, em quantidade e qualidade, acompanhando 0 crescimento dos indicadores socioeconômicos.

Sendo o crescimento populacional uma variável correlacionada ao consumo de água, cita-se ainda, o setor agrícola a nível mundial, o qual é responsável por $70 \%$ do uso da água, quando comparado aos setores comerciais e industriais (incluindo o setor energético) (WWAP, 2012). Segundo MAKRIDAKIS et al. (1998), é nesse contexto que se estabelece a necessidade do planejamento e previsão, identificando fatores quantitativos de gestão, propiciando o aproveitamento do tempo, tornando-o peça fundamental na tomada de decisões. A previsão é uma parte integrante do processo de tomada de decisão ao realizar atividades de gestão, pois verifica-se o aumento da necessidade ao tentar diminuir a dependência do acaso e tornar mecanismos gestores mais científicos ao lidar com o ambiente.

Com o objetivo de quantificar as previsões realizadas, utilizando dados históricos registrados sequencialmente no decorrer do tempo, utiliza-se o conceito de série temporal. Segundo MORETTIN \& TOLOI (2004), série temporal é uma sequência de observações coletadas no tempo, de forma igualmente espaçadas. As técnicas de análise dessas séries estão interessadas na avaliação de dependência dos dados, e requerem um desenvolvimento de modelos estocásticos e/ou dinâmicos, tendo diferentes áreas de aplicação, como a previsão de valores futuros da série, recorrendo a valores passados, examinando as interrelações entre uma ou mais variáveis (série multivariada), identificadas como forma de gerar um modelo matemático representativo dessas variáveis em estudo, no decorrer do tempo (BOX et al., 2016).

Aplicando esse conceito na previsão da demanda de água, tem-se uma expectativa de ferramenta eficaz na projeção de políticas públicas que corroborem para o desenvolvimento sustentável. Segundo a Agência Nacional de Águas (ANA), a água é um forte condicionante na desenvoltura social e econômica do país (ANA, 2010). Diante do exposto, esse trabalho busca avaliar de forma comparativa o desempenho de técnicas de séries temporais (suavizações exponenciais, ARIMA e algumas combinações) e objetiva ajustar um modelo matemático que explique a variação do consumo de água em um município no Sul do Brasil, tendo por base a análise dos dados históricos e utilizando o processo de modelagem via software estatístico livre. Espera-se que a adequação por meio do modelo matemático apresente previsões com erros aceitáveis.

\section{REFERENCIAL TEÓRICO}

Muitos métodos de previsão são baseados na concepção da existência de um padrão intrínseco no conjunto de dados que podem ser distinguidos dos valores aleatórios por suavização dos valores passados. O efeito dessa suavização é para eliminar a aleatoriedade e projetar valores futuros por meio dos padrões identificados usando previsões. O padrão da série é decomposto em padrões cíclicos e padrões sazonais, sendo cada componente identificado separadamente. Quanto melhor a identificação desses padrões mais acuradas serão as previsões (HYNDMAN \& ATHANASOPOULOS, 2016).

Os métodos usuais de decomposição identificam três componentes formadores de uma série $\left(Y_{t}\right)$, são elas: tendência, sazonalidade e ciclo. A tendência $\left(T_{t}\right)$ existe quando há um acréscimo ou decréscimo a longo prazo no nível da série. A sazonalidade $\left(S_{t}\right)$ ocorre quando a série é influenciada por fatores sazonais (bimestre, trimestre, semestre ou anuênio, por exemplo), ou seja, período de tempo 
fixado o qual imprimi uma característica padrão a variável. O ciclo $\left(C_{t}\right)$ ocorre quando os dados exibem variações crescentes ou decrescentes em períodos não fixados. Geralmente o ciclo apresenta períodos maiores que a sazonalidade. Os dados podem ser modelados conforme a equação 1 (MORETTIN \& TOLOI, 2004).

$$
Y_{t}=f\left(T_{t}, S_{t}, C_{t}, e_{t}\right)
$$

$A$ adição do componente erro $\left(e_{t}\right)$, conforme a equação 2 , chamada também de componente aleatória, ocorre devido a diferença entre o efeito combinado entre os padrões da série e seus valores atuais.

$$
e_{t}=Y_{t}-\hat{Y}_{t}
$$

na qual $\hat{Y}_{t}$ representa os valores previstos pelo modelo matemático.

A decomposição da série em estudo inicia-se verificando a existência das componentes geradoras da série. A aplicação adequada dos modelos baseado na significância estatística das componentes (tendência e sazonalidade) ocorre por meio de testes paramétricos e não-paramétricos. Os testes paramétricos possuem como condição a sua aplicação a distribuição normal dos dados para que os estimadores sejam eficientes. Na prática nem sempre está condição é verificada devido ao tamanho da amostra. Nesse caso, a busca por informações na própria variável em estudo, ocasiona possíveis correlações entre os dados. Por outro lado, a utilização dos testes não-paramétricos permite testar a significância dos dados dispensando a condição de normalidade da distribuição de probabilidade da variável em análise embora sejam mais fracos (estimadores menos eficientes) em relação aos paramétricos (AREAL \& ARMADA, 1999).

Segundo MORETTIN \& TOLOI (2004), dependendo das hipóteses feitas sobre a função $Y_{t}=f\left(T_{t}, S_{t}, C_{t}, e_{t}\right)$ tem-se duas classes de modelos: modelos de suavização exponencial e modelos autorregressivo. Nos modelos de suavização exponencial, essa função é determinada e erros $\left(e_{t}\right)$ é uma sequência aleatória, independente dessa função, não sendo correlacionados. A variável aleatória $e_{t}$ é caracterizada com média zero e variância constante. Na prática a hipótese da não correlação entre essa função e os erros introduz limitações ao modelo. De acordo com BOX et al. (2016) provavelmente nenhum fenômeno é totalmente determinístico por haver fatores estocásticos, como variações climáticas por exemplo, que podem influenciar nesse modelo. O quadro 1 apresenta modelos de suavização exponencial clássicos apropriados para a previsão de acordo com as componentes identificadas em uma série (MUN, 2010).

\begin{tabular}{|l|l|l|}
\multicolumn{2}{|l}{ QUADRO 1 - Métodos Clássicos de série temporal } \\
\hline \multirow{2}{*}{ SEM TENDÊNCIA } & \multicolumn{1}{|c|}{ SEM SAZONALIDADE } & \multicolumn{1}{c|}{ COM SAZONALIDADE } \\
\cline { 2 - 3 } & Sédia Móvel Simples & Sazonal Aditivo \\
\cline { 2 - 3 } & Suavização Exponencial Simples & Sazonal Multiplicativo \\
\cline { 2 - 3 } COM TENDÊNCIA & Média Móvel Dupla & Aditivo de Holt-Winters \\
\cline { 2 - 3 } & Suavização Exponencial Dupla & Multiplicativo de Holt-Winters \\
\hline
\end{tabular}

Fonte: MUN (2010).

Como uma série temporal tem seus valores coletados ao longo do tempo, espera-se que seus dados possuam uma correlação seriada. A metodologia BoxJenkins, genericamente conhecidos por modelos ARIMA (Auto Regressive Integrated Moving Average), possuindo um acrônimo na literatura em português por Autorregressivo Integrado de Médias Móveis, são modelos matemáticos que 
permitem verificar o comportamento desta correlação seriada ou autocorrelação entre os valores da série temporal, e com base no seu respectivo comportamento apresentar predições futuras. O modelo quando bem ajustado permite uma boa aproximação, embora modelos de ordens diferentes possam fornecer resultados aceitáveis (WERNER \& RIBEIRO, 2003). Segundo FAVA (2000), os modelos ARIMA resultam da combinação de três componentes, denominados "filtros": o componente autorregressivo (AR), o filtro de integração (I) e o componente de média móvel (MA). Uma série pode ser modelada por esses filtros ou por apenas um subconjunto deles.

Os modelos ARIMA são definidos quanto a sua estacionaridade. O processo será fracamente estacionário quando o seu valor esperado não depender de um momento preciso, quando a variância dos seus valores independente do período seja constante e finita e a covariância entre diferentes observações depender do intervalo entre essas observações, mas não do período em que as tomamos (ANDRADE, 2004). Segundo esse autor, os processos estacionários da metodologia Box-Jenkins, são basicamente três: autorregressivo de ordem $A R(p)$, médias móveis de ordem $M A(q)$, autorregressivo de médias móveis de ordem ARMA(p, q).

Nos processos não-estacionários assume-se que a série é não estacionária por ocorrer tendência e/ou outros padrões que alteram o nível da série. Um dos caminhos para remover a não-estacionaridade é utilizar o método de diferenciação que busca subtrair uma observação $Y_{t}$ de seu valor anterior $Y_{t-1}$. A quantidade de diferenciações representa a ordem do filtro de integração, I(d). Os processos autoregressivos integrados de médias móveis de ordem $\operatorname{ARIMA}((p, d, q)$ e os autoregressivos integrados de médias móveis sazonais, de ordem SARIMA(P, D, $Q$, $p, d, q)_{s}$, com estação sazonal $s$, utiliza primeiramente o filtro de integração $l(d)$ removendo a não estacionaridade seguido da aplicação dos filtros $A R(p)$ e $M A(q)$.

De acordo com MAKRIDAKIS et al. (1998) na previsão, a precisão é tratada como critério crucial para a seleção de um método de previsão. A precisão pode ser obtida por medidas de acurácia baseada na diferença entre o real valor e o valor predito. Segundo HYNDMAN \& ATHANASOPOULOS (2016), as duas medidas de acurácia mais comumente utilizadas as quais são dependentes de escalas são baseadas em erros absolutos ou erros quadráticos, sendo apresentadas nas equações 3 e 4.

$$
\begin{gathered}
\text { Mean absolute error: } M A E=\text { média }\left(\left|e_{t}\right|\right), \\
\text { Root mean squares error: } R M S E=\sqrt{\operatorname{média}\left(e_{t}^{2}\right)}
\end{gathered}
$$

em que $e_{t}$ é apresentado conforme a equação 2 .

Já o erro percentual médio absoluto (MAPE), conforme equação 5 , tem a vantagem de ser independente de escala, utilizado para a comparação entre conjuntos de dados diferentes.

$$
\text { Mean absolute percentage error: } M A P E=\text { média }\left(\frac{100 e_{t}}{Y_{t}}\right)
$$

Ao comparar os métodos de previsão em um único conjunto de dados, o MAE é prático e de fácil entendimento. Na literatura, verifica-se o uso do Mean Absolute Perceptual Error (MAPE) além do MAE e RMSE. Estes serão os indicadores de acurácia utilizados para a série estudada. 
Ainda com o objetivo de uma modelagem matemática que apresente uma acurácia maior, pode-se realizar combinações dos resultados de diferentes modelos, pois cada técnica empregada não permite a incorporação completa de todos as informações incluídas no processo (WERNER \& RIBEIRO, 2006). Para tratar esta lacuna, é necessário incorporar diversas previsões em uma nova e única previsão propiciando diminuir os erros buscando valores preditos mais acurados (ARMSTRONG, 2001; WERNER, 2005).

Nesse trabalho utiliza-se métodos objetivos de combinação, os quais fazem uso da matemática, originários pelo estudo de Bates e Granger (1969). Os referidos autores utilizaram métodos de combinação linear de duas previsões não viesadas em que para a combinação é concedida um peso para cada uma das previsões individuais. A estrutura seguida é a exposta na equação 6:

$$
F_{c}=w F_{1}+(1-w) F_{2}
$$

em que: $w$ é o peso atribuído a previsão e $F_{1}$ e $F_{2}$ são as previsões individuais a serem combinadas.

A maneira mais simples de combinar previsões é a realização da média aritmética em que $w=0,5$ demonstrando mais acurácia dentre os métodos individuas mais elaborados. Analisando ainda o desempenho de métodos de previsão individuais disponíveis, podemos por meio deles atribuir pesos maiores aos que apresentaram maior desempenho, e utilizando os indicadores de erros permitir que 0 sistema de ponderação se adapte ao longo do tempo, uma vez que o desempenho possa modificar-se. Atendendo as condições necessárias citadas, a atribuição de pesos para cada previsão baseia-se na proporção inversa à soma do indicador de erro das respectivas previsões individuais os quais trabalham com erros absolutos, entre eles o MAPE (equação 7), MAE (equação 8) e RMSE (equação 9) e/ou MSE (NEWBOLD \& BOS, 1994).

$$
\begin{aligned}
w_{M A P E} & =\frac{1 / M A P E_{1}}{1 / M A P E_{1}+1 / M A P E_{2}} \\
w_{M A E} & =\frac{1 / M A E_{1}}{1 / M A E_{1}+1 / M A E_{2}} \\
w_{R M S E} & =\frac{1 / R M S E_{1}}{1 / R M S E_{1}+1 / R M S E_{2}}
\end{aligned}
$$

Além da média aritmética, tem-se a média geométrica e harmônica que serão empregadas na combinação entre os modelos de suavização exponencial e ARIMA (BOX et al., 2016).

\section{MATERIAL E MÉTODOS}

De acordo com BERTRAND \& FRANSOO (2002), esta investigação pode ser classificada como pesquisa aplicada, tendo um estudo de caso, no qual o pesquisador busca criar um modelo que se adeque a descrição das relações causais que possam existir na realidade. Isto conduz para o entendimento do corrente processo podendo assim promover a compreensão da realidade estudada. A abordagem para o problema é quantitativa e o modelo de pesquisa matemática tomará como base a Tabela 1 e a Figura 1. 


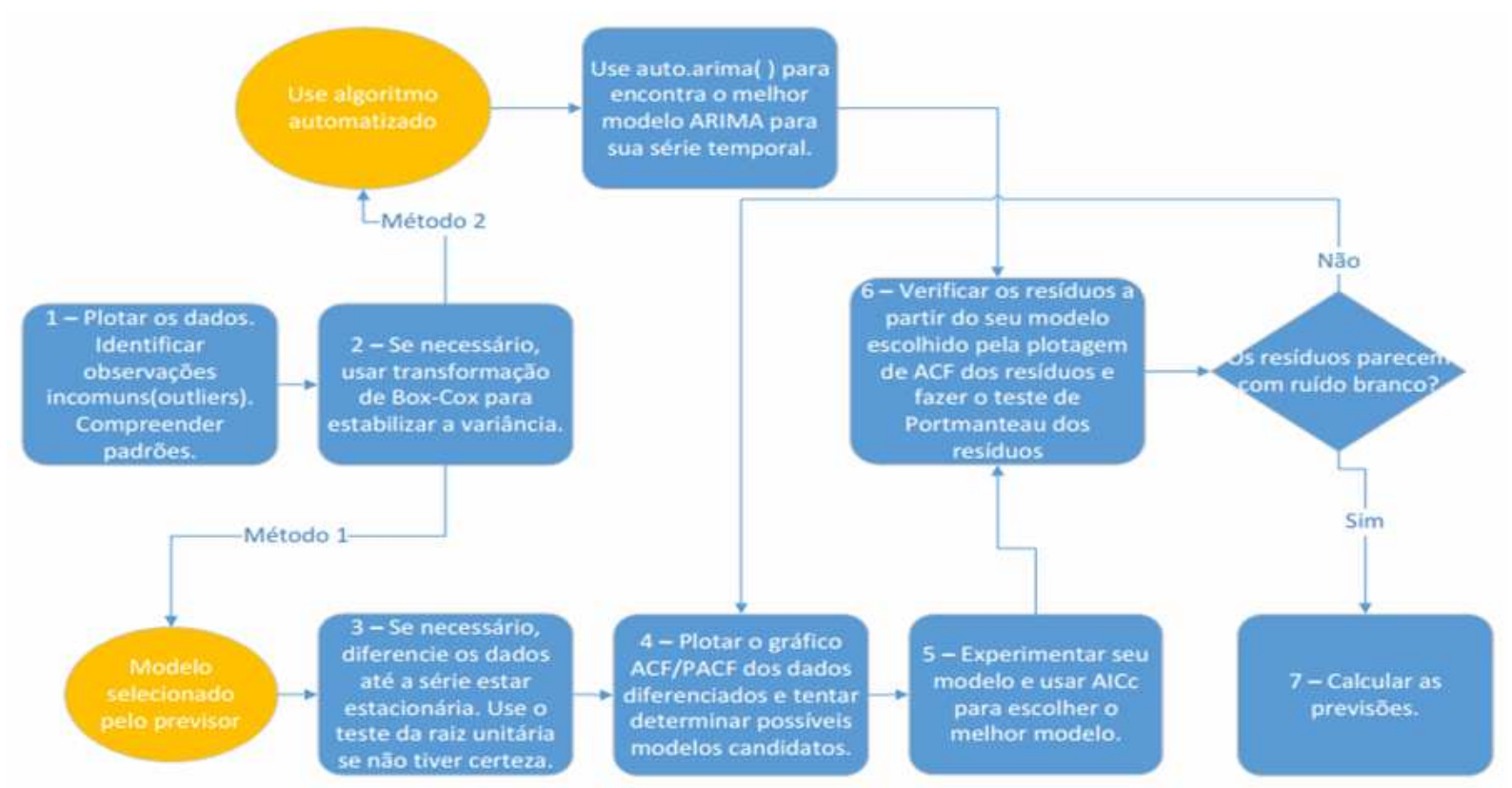

FIGURA 1 - Processo geral para a previsão com base num modelo ARIMA

Fonte: Adaptado de HYNDMAN \& ATHANASOPOULOS (2016).

Emprega-se o método 2 da Figura $1 \mathrm{com} 0$ algoritmo baseado na metodologia de HYNDMAN \& KHANDAKAR (2008), tendo a função auto.arima() encontrado no package forecast do software $R$. A função utilizada possui parâmetros configuráveis que possibilita obter modelos mais otimizados. Com o objetivo de testar as aproximações oferecidas pelo algoritmo testou-se duas configurações intituladas "modelo SARIMA - com aproximações" e "modelo SARIMA - sem aproximações". Ao se aplicar o modelo com aproximações, manteve-se as configurações padronizadas. Já no modelo sem aproximações buscou-se estabelecer o máximo de detalhes possíveis.

Além disso, adotou-se a seguinte estratégia: (i) os critérios de informação (AIC: Akaike information criterion; BIC: Bayesiano information criterion; e AICc: Akaike information criterion corrected) para seleção mais parcimoniosa do modelo, percorreu-se todos os modelos possíveis pelo espaço de estados, buscando um melhor ajuste sazonal; e (ii) os testes de raiz unitária utilizados foram KPSS (Kwiatkowski-Phillips-Schmidt-Shin), ADF (Augmented Dickey-Fuller) e PP (PhillipsPerron) e evitou-se aproximações por meio da condição do somatório dos erros quadráticos e critérios de informações.

A cidade escolhida para o estudo de caso, localiza-se na região Sul do Brasil e tem uma população estimada em torno de 80 mil habitantes (IBGE, 2015). Para isso, foram utilizados dados sobre o volume de água captado mensalmente no período de janeiro de 2000 a dezembro de 2011. Esses dados foram fornecidos por meio do banco de dados da companhia de saneamento do município, a qual possui a concessão para operar o sistema de abastecimento de água e saneamento básico. A série é univariada e está organizada conforme ilustra a Figura 2.

A variável analisada foi o volume de água mensal captado no município, no período de janeiro de 2000 a dezembro de 2011 totalizando, assim, uma série temporal com 144 amostras. Os valores mensais do ano de 2011 foram reservados para comparação com as previsões dos modelos ajustados, restando 132 amostras para o processo de modelagem. As análises dos dados foram feitas utilizando a plataforma R, um ambiente de software livre para computação estatística e gráficos 
(R DEVELOPMENT CORE TEAM, 2016). Empregou-se a interface RStudio por possuir uma workspace amigável para o trabalho e apresentar pacotes (add-ons) com diversas ferramentas estatísticas que facilitam a resolução do problema em questão (RSTUDIO, 2015). Para a realização do estudo de caso procedeu-se a elaboração de um algoritmo computacional na referida plataforma.

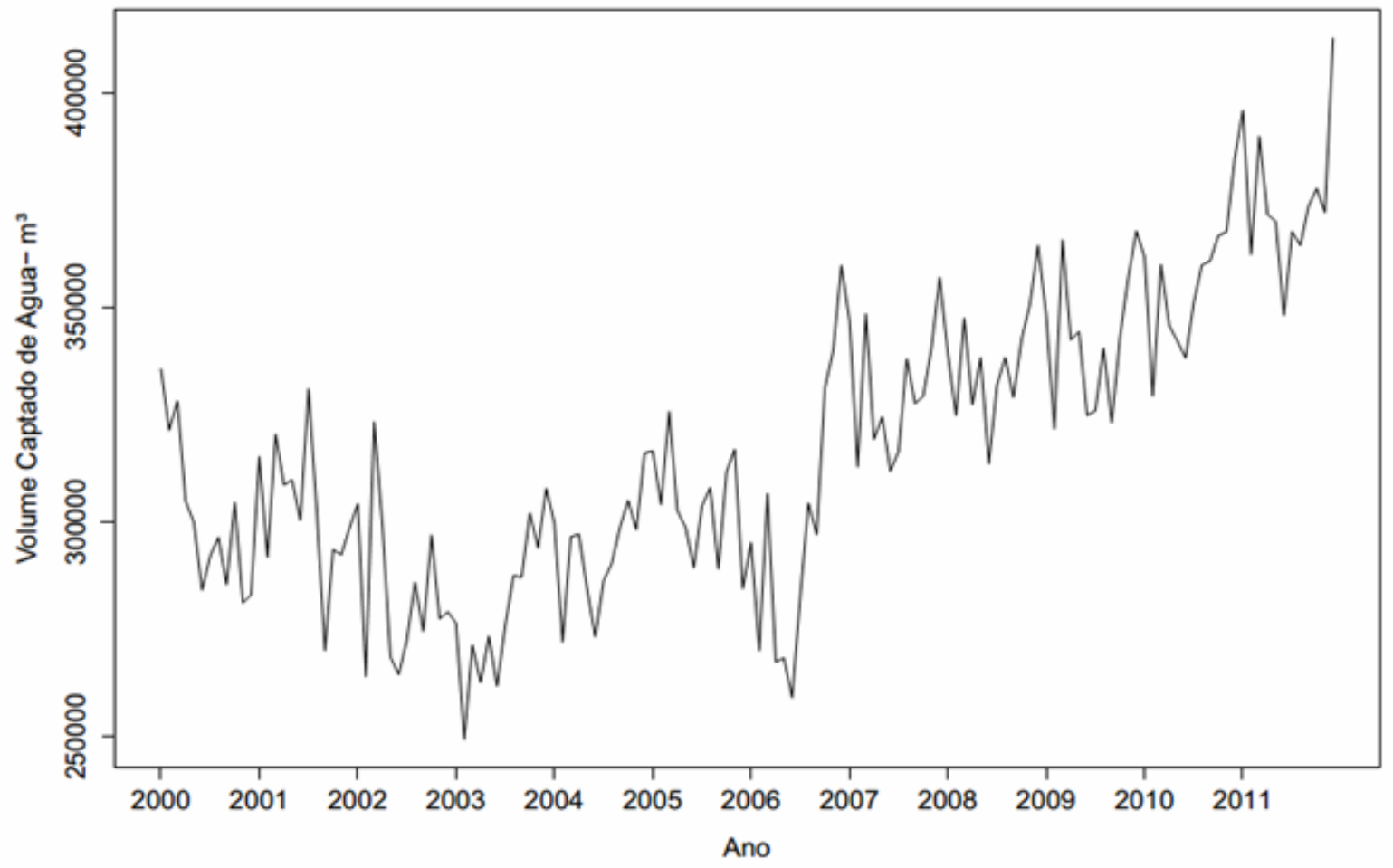

FIGURA 2 - Série do volume de água captado no município em estudo (2000-2011)

$O$ processo de modelagem computacional via software $R$, foi executado em três etapas, são: (i) verificação, aplicação e previsão por intermédio dos modelos individuais (suavização exponencial e ARIMA); (ii) combinação entre as melhores previsões dos modelos da primeira etapa; e (iii) avaliação das medidas de acurácia entre os modelos individuais e suas combinações.

$\mathrm{Na}$ primeira etapa, da modelagem de séries temporais, foi feita a inspeção gráfica para identificar as características importantes da série como tendência, sazonalidade, variabilidade, observações atípicas (outliers) dentre outros. Com isto faz-se necessário a verificação dos possíveis padrões formadores da série antes de aplicar qualquer procedimento para a estimação (MORETTIN \& TOLOI, 2004). Sabendo que a análise empírica é uma condição necessária, porém não suficiente para descrição do modelo, utiliza-se os testes não-paramétricos (AREAL \& ARMADA, 1999). Para a tendência os testes utilizados foram Cox - Stuart e MannKendall. Por outro lado, para a sazonalidade os testes utilizados foram KruskalWallis e Friedman. Mediante a análise dos testes escolhe-se o melhor modelo de acordo com a Figura 1.

Após a análise dos testes não-paramétricos e a escolha do modelo de suavização exponencial, aplicam-se os modelos Autoregressivos (AR), Integrados (I) de Médias Móveis (MA), ARIMA, derivados da metodologia Box-Jenkins. A modelagem ARIMA no $R$ utiliza uma variação do algoritmo de HYNDMAN \& KHANDAKAR (2008) que combina testes de raiz unitária, minimização do AICc (Akaike information criterion Corrected) e Estimativa de Máxima Verossimilhança (maximum likelihood estimation - MLE) para obter o modelo ARIMA. O algoritmo 
segue as seguintes etapas: (i) o número de diferença d é determinado por meio de repetidos testes KPSS; e (ii) os valores de $p$ e $q$ são escolhidos por meio da minimização do AICc após diferenciação dos dados d vezes. Ao invés de considerar todas as combinações o algoritmo testa modelos adjacentes como forma de passar pelo espaço de estados até encontrar o menor AICc dentre eles.

Escolhido o modelo de suavização e o modelo ARIMA realiza-se a previsão da série com até 12 passos à frente quanto ao horizonte de previsão, determinando os valores mensais para o ano 2011. As medidas de acurácia (RMSE, MAE e MAPE) foram calculadas de ambas as previsões.

$\mathrm{Na}$ segunda etapa aplicaram-se técnicas de combinações que forneceram novos resultados de previsões e medidas de acurácia. Por fim procedeu a análise dos estimadores de acurácia para a identificação do modelo que apresentar o menor erro de previsão.

\section{RESULTADOS E DISCUSSÃO}

O primeiro passo da análise de uma série temporal é a construção do gráfico, o qual poderá revelar características importantes (HYNDMAN \& ATHANASOPOULOS, 2016). A série do volume captado de água desse município, pode ser visualizada na Figura 2.

Alguns pontos podem ser percebidos por análise empírica (inspeção visual): (i) a série apresenta uma quebra na tendência nos anos de 2003 e 2006; (ii) entre 2003 e 2006 houve um crescimento (2003-2005) acompanhando de uma queda (2005-2006) na tendência; (iii) a partir de 2006 apresentou um comportamento de tendência com crescimento mais uniforme, diferentemente dos intervalos 2000-2003 e 2003-2006, provavelmente causados por fatores como crescimento populacional; e (iv) é possível verificar a existência de certa sazonalidade nos dados, que pode ter sido causada por fatores como estações climáticas bem definidas.

A adoção de testes não-paramétricos não exige que os dados obedeçam ao critério de distribuição normal dos dados. Permite assim que os estimadores não sejam viesados e assim representar de maneira formal a possível existência de padrões como tendência e sazonalidade o qual é apresentado na Tabela 1.

TABELA 1 - Testes Não-Paramétricos para a série em estudo

\begin{tabular}{c|c|c|c}
\hline \multicolumn{5}{c}{ TENDÉNCIA } \\
\hline \multirow{2}{*}{ Teste } & Hipótese Nula & Hipótese Alternativa $(\mathbf{p}<5 \%)$ & $\mathbf{p}$-valor \\
\hline \multirow{4}{*}{ Mantuart } & Aleatório & Não-aleatório & $1,1828 \times 10^{-9}$ \\
\cline { 2 - 4 } & Aleatório & Tendência Decrescente & 1 \\
\cline { 2 - 4 } & Aleatório & Tendência Crescente & $5,9139 \times 10^{-10}$ \\
\hline \multicolumn{4}{|c}{ SAZONALIDADE } \\
\hline Kruskal-Wallis & Não há Sazonalidade & Há Sazonalidade & $1,5112 \times 10^{-14}$ \\
\hline Friedman & Não há Sazonalidade & Há Sazonalidade & $7,9596 \times 10^{-16}$ \\
\hline
\end{tabular}

Fonte: Elaborado pelos autores.

O teste Cox-Stuart utiliza três configurações possibilitadas pela implementação computacional no R. As configurações com a hipótese alternativa "não-aleatório" e "tendência crescente", apresentaram o p-valor muito menor que $1 \%$ que, de acordo com o teste, configura o aparecimento de tendência crescente. $O$ teste Mann-Kendall sazonal verificou o aparecimento de tendência com p-valor muito menor que $1 \%$. Nos testes de sazonalidade de Kruskal-Wallis e Friedman, ambos obtiveram p-valor menores que $1 \%$ verificando como resposta a hipótese alternativa dos testes. Isso evidencia o aparecimento de sazonalidade e a tendência crescente 
na série em análise. Comparando a análise empírica e a Tabela 2, a tendência decrescente é menos significativa na formação da série comparada a tendência crescente.

A análise da série por meio de testes não-paramétricos permite avaliar de maneira mais direta os modelos que melhor se adequam a série. Com isso identificou-se na Tabela 1 que os modelos possíveis são o Aditivo Holt-Winters e Multiplicativo Holt-Winters em que os termos aditivo e multiplicativo refere-se à sazonalidade com amplitude constante ou variando em relação a tendência, respectivamente.

A partir dos dados foi utilizada função "hw" disponível no package ses\{forecast\} (HYNDMAN \& KHANDAKAR, 2008) no software $R$ para realizar 0 ajuste da suavização exponencial de Holt-Winters do volume de água. Foram determinadas as constantes de suavização alfa $(\alpha)$, beta $(\beta)$ e gama ( $\gamma$ ) e as medidas de acurácia do modelo aditivo e multiplicativo de Holt-Winters. Ao realizar os cálculos computacionais, os gráficos foram plotados com a suavização e previsões para o ano de 2011 (previsão de até 12 passos à frente) com intervalo de 95\% de confiança conforme ilustram as Figura 3 e 4.

O modelo de suavização exponencial Holt-Winters com sazonalidade aditiva e multiplicativa foi aplicado e apresentou um bom ajuste e previsão. $O$ destaque veio do modelo de sazonalidade multiplicativo demonstrando que a série em estudo apresenta variação sazonal proporcional ao seu nível. Este modelo tem boa aplicabilidade para séries com alta sazonalidade. As Figuras 3 e 4 apresentaram o resultado das previsões de forma visual e é possível destacar que a série multiplicativa apresenta um crescimento mais acentuado e nesse caso pelas medidas de acurácia (MAE, RMSE e MAPE) utilizando como base de comparação os dados de validação e as constantes de suavização gerou o modelo matemático adequado ao comportamento da série.

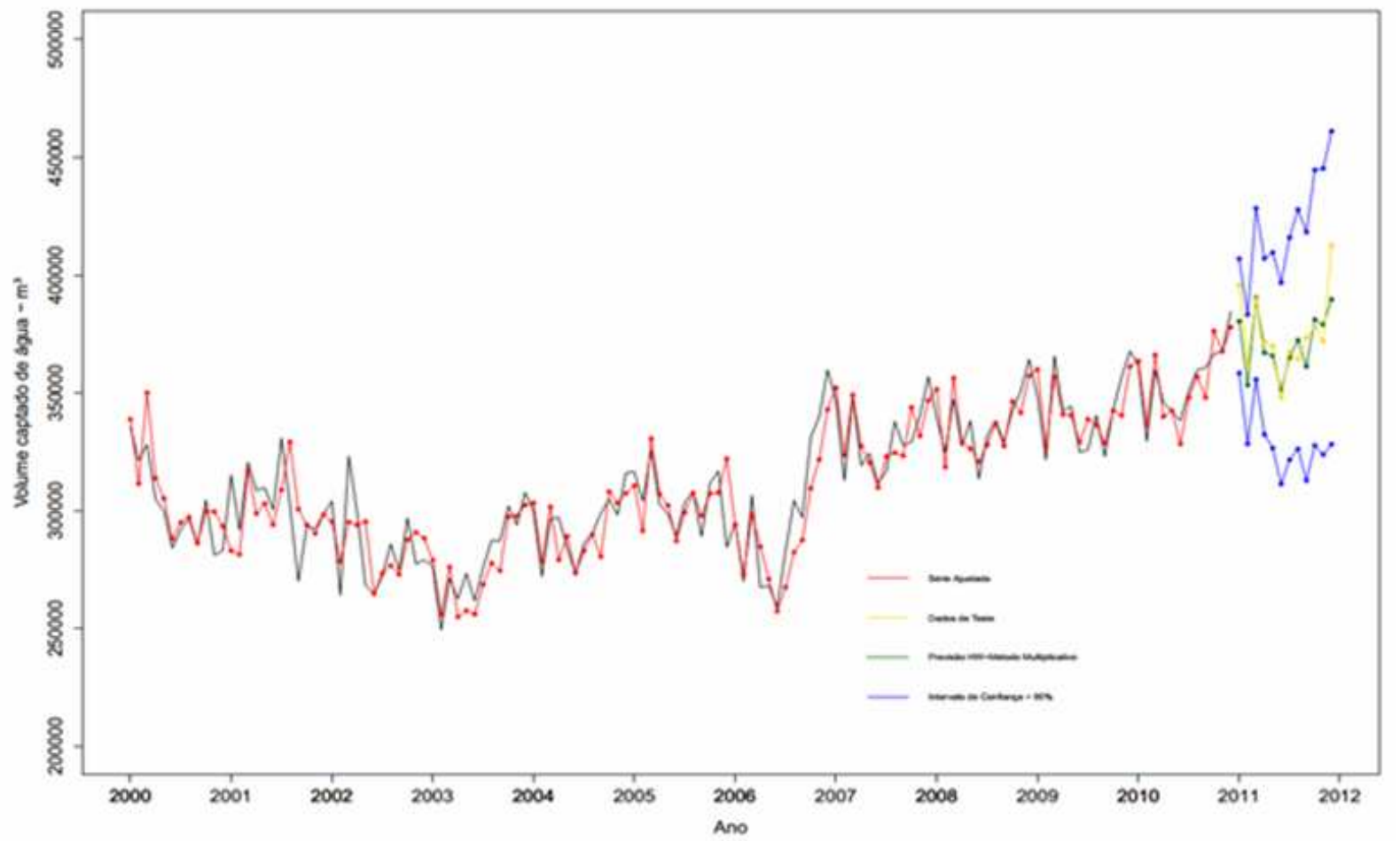

FIGURA 3 - Resultado do ajuste e previsão da série por Holt-Winters método aditivo 


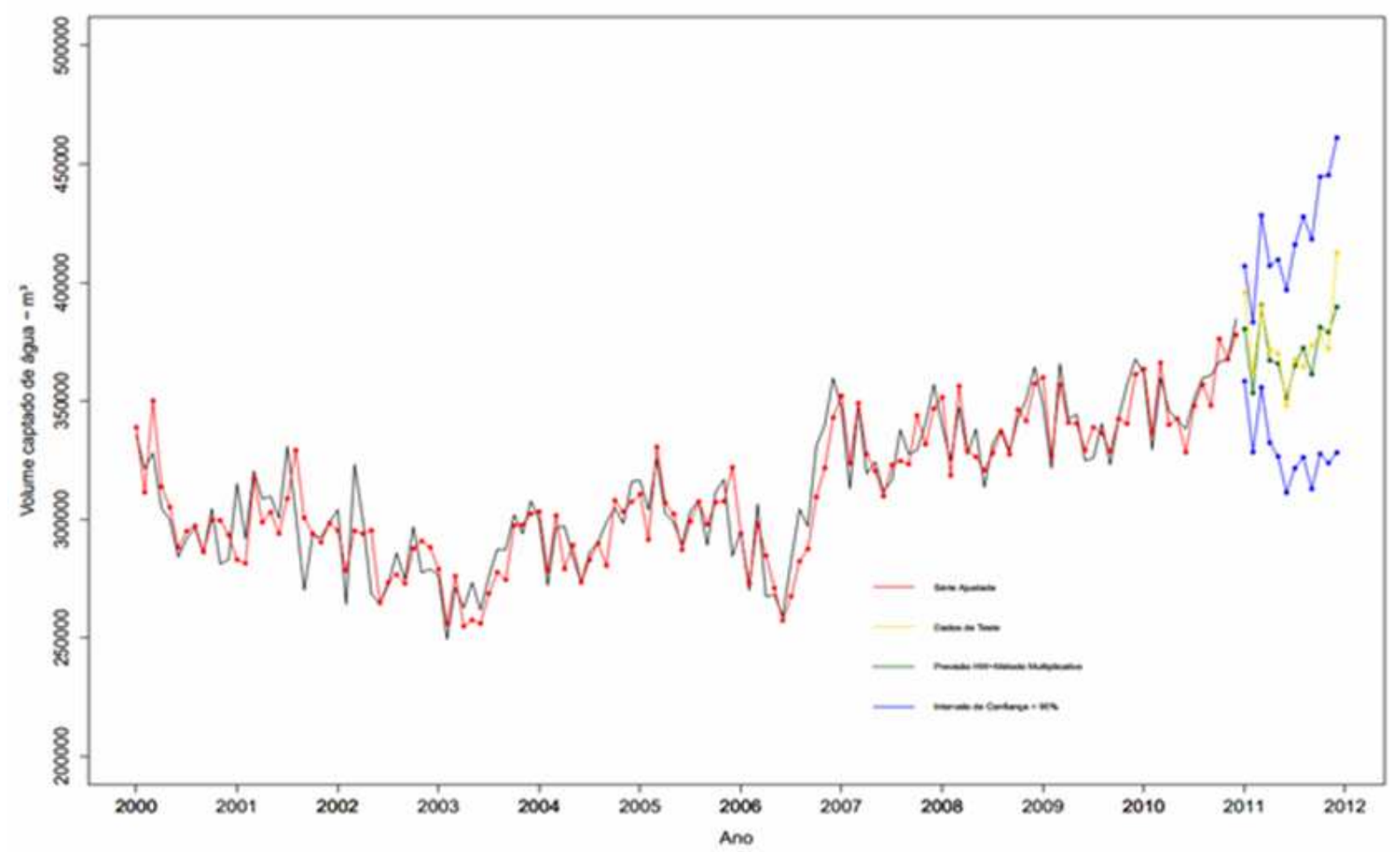

FIGURA 4 - Resultado do ajuste e previsão da série por Holt-Winters método multiplicativo

As constantes de suavização foram calculadas de modo que a soma do quadrado dos erros de suavização fossem consideradas mínima. As constantes encontradas para o modelo Aditivo de Holt-Winters (HWa) foram: $\alpha=0,7202, \beta=$ $1 \times 10^{-4}$ e $\gamma=2 \times 10^{-4}$. Já para o modelo Multiplicativo de Holt-Winters (HWm), as constantes foram: $\alpha=0,7081, \beta=0,056$ e $\gamma=1 \times 10^{-4}$. Na Tabela 4, segue as medidas de acurácia utilizadas para verificar se os valores previstos estão consonantes com o conjunto dados de teste.

Seguindo o método 2 da metodologia empregada, aplicou-se os testes estatísticos de Box-Pierce e de Ljung-Box para examinar os resíduos, os quais são conhecidos como testes "portmanteau" (R DOCUMENTATION, 2015). O teste é aplicado aos resíduos após a montagem do modelo ARIMA que examina as $m$ autocorrelações dos resíduos. Os resultados encontrados estão postos na Tabela 2.

Aplicado o teste portmanteau, o $\mathrm{p}$-valor encontrado para o teste de ambos os modelos previstos válida a hipótese nula. Realizando a previsão por meio da função implementada, retorna os resultados apresentados nas Figura 5 e 6 . Os modelos ajustados possuem os coeficientes que se seguem:

SARIMA $(0,1,1)(2,0,0)_{12}-$ Modelo sem Aproximações: $\left(\theta_{1}=-0,3575, \Phi_{1}=0,3707, \Phi_{2}=0,3461\right)$ SARIMA $(2,1,0)(2,0,0)_{12}$-Modelo com Aproximações: $\left(\square_{1}=-0,3240, \square_{2}=-0,1726, \Phi_{1}=0,3770, \Phi_{2}=\right.$ $0,3500)$

TABELA 2 - Análise de Resíduos pelo Teste de Portmanteau

\begin{tabular}{|c|c|c|c|c|}
\hline \multicolumn{5}{|c|}{ Resíduos } \\
\hline $\begin{array}{c}\text { Teste } \\
\text { "Portmanteau" }\end{array}$ & $\begin{array}{l}\text { Hipótese } \\
\text { Nula }\end{array}$ & $\begin{array}{c}\text { Hipótese } \\
\text { Alternativa } \\
(p<5 \%)\end{array}$ & \begin{tabular}{c}
\multicolumn{1}{c}{ Sem } \\
aproximação \\
p
\end{tabular} & $\begin{array}{l}\text { Com } \\
\text { aproximação } \\
\text { or }\end{array}$ \\
\hline $\begin{array}{l}\text { Box-Pierce } \\
\text { Ljung-Box }\end{array}$ & $\begin{array}{l}\text { O modelo não exibe } \\
\text { falta de ajuste }\end{array}$ & $\begin{array}{l}\text { O modelo exibe } \\
\text { falta de ajuste. }\end{array}$ & $\begin{array}{l}0,08812 \\
0,06014\end{array}$ & $\begin{array}{c}0,09849 \\
0,0686\end{array}$ \\
\hline
\end{tabular}




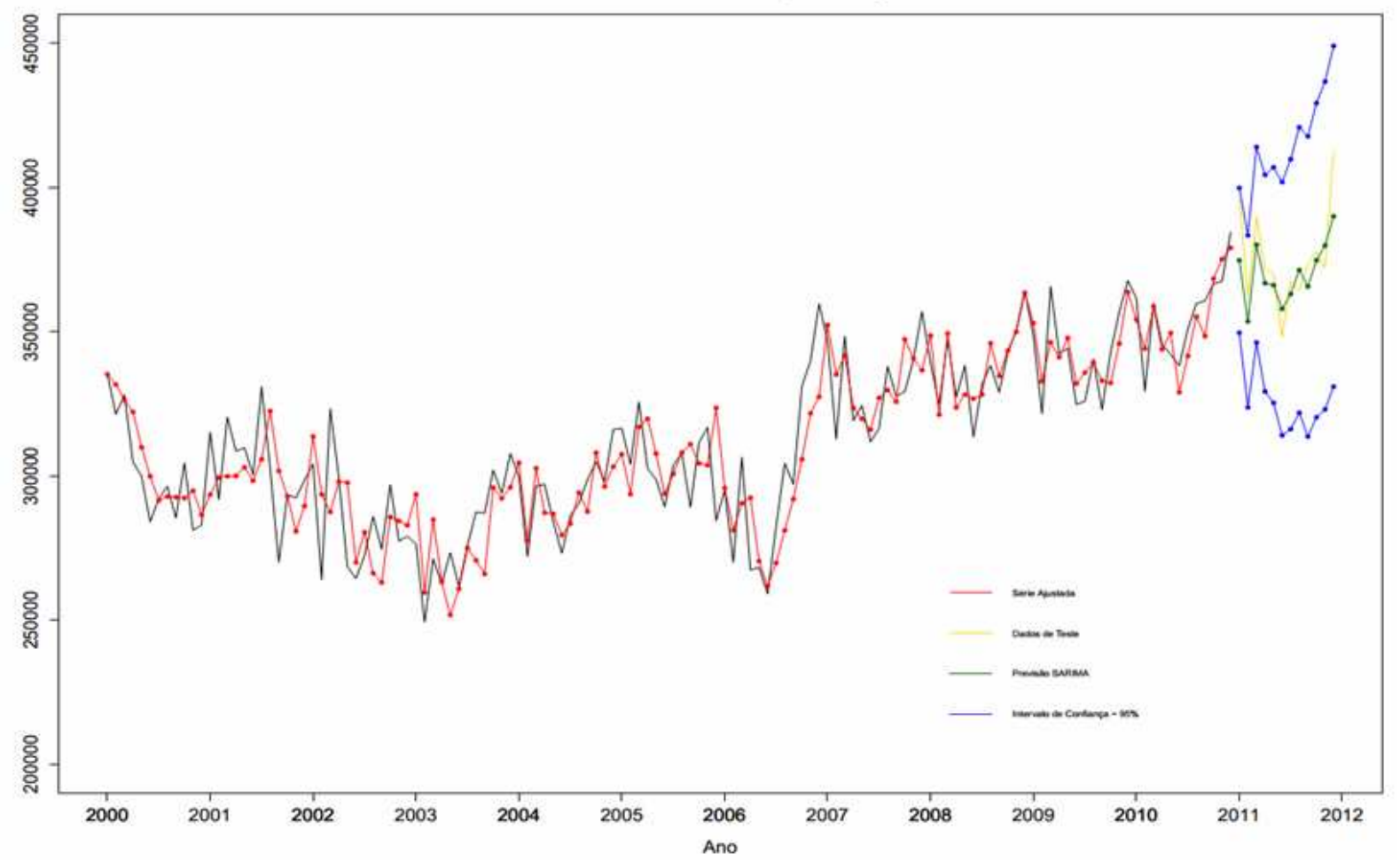

FIGURA 5 - Resultado do ajuste e previsão: $\operatorname{SARIMA}(0,1,1)(2,0,0)_{12}$

A implementação dos modelos ARIMA quanto a ordem dos filtros $A R(p)$, MA(q) e I(d) foram calculados por meio da manipulação dos parâmetros do algoritmo sugerido por HYNDMAN \& KHANDAKAR (2008). Foram utilizadas duas configurações das disponíveis. Ambos os modelos apresentaram o filtro I(1) demonstrando que a série é não-estacionária, reafirmando o resultado dos testes não-paramétricos quanto as características da série. A inspeção dos resultados de forma visual não proporcionou certeza quanto a melhor qualificação entre os modelos, entretanto pelos indicadores MAE, RMSE e MAPE o modelo ARIMA sem aproximação surge como melhor opção. $O$ modelo foi validado por meio da análise de resíduos descrito pelos testes de portmanteau em que a hipótese nula considerando um intervalo de confiança de $95 \%$ não aponta falta de ajuste.

Diferente dos modelos Holt-Winters, os ARIMAs apresentaram diferença percentual menor que $1 \%$ entre os respectivos indicadores de erro. As modificações empregadas nos parâmetros computacionais não tiveram uma interferência significativa no que tange a melhora do modelo. Contudo, utilizou-se os melhores modelos de cada classe de acordo com a acurácia e foram obtidas as combinações.

De forma similar aos resultados apresentados para os modelos de HW, a Tabela 3 também destaca as medidas de acurácia dos modelos SARIMAs. $\mathrm{Na}$ sequência, utilizou-se o melhor modelo de HW e o melhor SARIMA para realizar as combinações com a média aritmética, geométrica, harmônica e com os pesos calculados inversamente aos indicadores: MAPE, MAE e RMSE. 


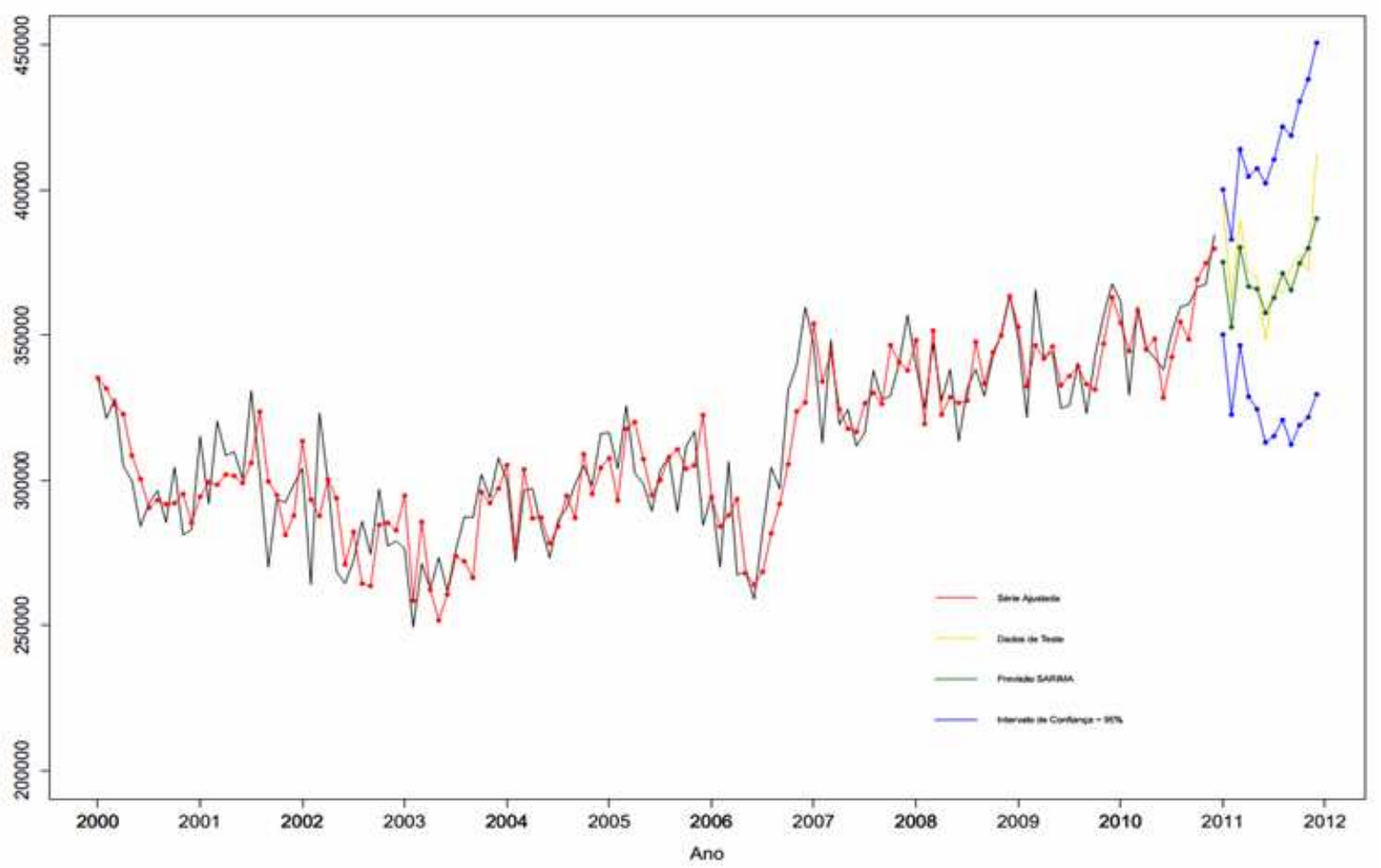

FIGURA 6 - Resultado do ajuste e previsão: ARIMA $(2,1,0)(2,0,0)_{12}$

TABELA 3 - Medidas de Acurácia dos Modelos de Suavização e SARIMA

\begin{tabular}{lcccr}
\hline \multicolumn{1}{c}{$\begin{array}{c}\text { Medidas de } \\
\text { Acurácia }\end{array}$} & $\begin{array}{c}\text { HW- } \\
\text { Aditivo }\end{array}$ & $\begin{array}{c}\text { HW - } \\
\text { Multiplicativo }\end{array}$ & $\begin{array}{c}\text { SARproximação } \\
\text { SAlaproximação }\end{array}$ \\
\hline RMSE & 12,39154728 & 10,2170415 & 11,01775183 & 10,95569393 \\
MAE & 9,078994843 & 8,542142271 & 9,204889846 & 9,22679773 \\
MAPE (\%) & 2,35254871 & 2,267647673 & 2,409705984 & 2,416954932 \\
\hline
\end{tabular}

Ambos os modelos apresentaram um ajuste e previsão visualmente razoável para série. O modelo multiplicativo obteve um MAPE de 2,27\% comparado ao MAPE de $2,35 \%$ do modelo aditivo, apresentando melhor ajuste e previsão entre os modelos de Holt-Winters. Os modelos SARIMAs não obtiveram diferença relevante, embora o modelo sem aproximação obtivesse o melhor MAPE de 2,41\%. Utilizando o modelo Multiplicativo Holt-Winters e o SARIMA sem aproximação realizou-se as combinações propostas. As respectivas medidas de acurácia dessas combinações encontram-se na Tabela 4.

TABELA 4 - Medidas de Acurácia das Técnicas de Combinação

\begin{tabular}{llllccc}
\hline $\begin{array}{c}\text { Medidas de } \\
\text { Acurácia }\end{array}$ & $\begin{array}{c}\text { Média } \\
\text { Aritmética }\end{array}$ & $\begin{array}{c}\text { Média } \\
\text { Geométrica }\end{array}$ & $\begin{array}{c}\text { Média } \\
\text { Harmônica }\end{array}$ & $\begin{array}{c}\text { Inverso } \\
\text { RMSE }\end{array}$ & $\begin{array}{c}\text { Inverso } \\
\text { MAE }\end{array}$ & $\begin{array}{c}\text { Inverso } \\
\text { MAPE }\end{array}$ \\
\hline RMSE & 9,832193 & 9,831761 & 9,831419 & 9,817052 & 9,817187 & 9,819815357 \\
MAE & 8,214149 & 8,207638 & 8,201128 & 8,223320 & 8,223232 & 8,221535896 \\
MAPE (\%) & 2,161723 & 2,159830 & 2,157938 & 2,164897 & 2,164867 & 2,164279787 \\
\hline
\end{tabular}

Ao comparar os erros dos previsores, verifica-se que o RMSE o qual trabalha com erros quadráticos, ponderando mais significativamente erros maiores apresentou um melhor ajuste para as combinações utilizando os pesos. Entretanto a diferença entre a melhor e a pior técnica das combinações de um respectivo indicador é menor que 1\%. Da mesma forma, utilizando o MAE e MAPE constata-se que as combinações por meio das médias apresentaram resultados mais promissores que as combinações com pesos inversos. A melhor combinação foi a média harmônica, seguido da média geométrica e, por fim, a média aritmética. 
As combinações foram acompanhadas do cálculo de médias (aritmética, geométrica e harmônica) e combinação linear tendo os pesos definidos pelas próprias medidas de acurácia. Como citado em diversas referências bibliográficas, as combinações nesse trabalho apresentaram melhor ajuste do que os métodos individuais. Ao calcular novas previsões partindo das previsões individuais possibilita incluir a esse novo valor predito características agrupadas de cada modelo individual respeitando o valor da ponderação no decorrer da série.

\section{CONCLUSÕES}

No presente estudo buscou-se realizar previsões de demanda quantitativas utilizando diferentes modelos de séries temporais e algumas técnicas de combinação dessas. Para verificar se havia diferença entre a acurácia das previsões individuais e suas combinações, foi utilizado a série do volume de água captado em um município do Sul do Brasil. Por inspeção visual, a série apresentou tendência e sazonalidade o qual foi confirmado pelos testes não-paramétricos aplicados. Utilizou-se modelos de suavização exponencial, SARIMA e 6 (seis) combinações das previsões individuais.

A série disponível para análise continha uma base de 11 anos (2000 a 2011), com dados registrados mensalmente, sendo o ano de 2011 reservado para o processo de validação. Para atingir esse objetivo foram transcorridas algumas etapas: (i) caracterização da série temporal, a qual confirmou tendência e sazonalidade por meio de testes não paramétricos; (ii) ajuste e comparação dos modelos de suavização exponencial aditivo e multiplicativo de Holt-Winters (HW), com destaque para o modelo multiplicativo; (iii) ajuste e comparação dos modelos SARIMA com e sem aproximação, sendo que esse último modelo foi o que se revelou mais acurado; e (iv) aplicação de 6 tipos de combinação dos melhores modelos HW e SARIMA, as quais apresentaram resultados mais acurados que os preditores individuais. Contudo, vale ressaltar que todos os modelos avaliados apresentaram resultados promissores, pois os MAPEs foram inferiores a $3 \%$.

O planejamento de curto prazo utilizado para o abastecimento de água demonstrou-se satisfatório tendo a empregabilidade de dois modelos com considerações diferentes, em relação ao tratamento dos dados, seguido da combinação desses valores. Por tratar-se de um trabalho quantitativo não foi feito ajustes qualitativos. Reconhece-se a aplicabilidade dos métodos como bons preditores, podendo ser utilizados pela empresa de saneamento. É importante ressaltar que os valores utilizados não incluem perdas do sistema por utilizar como base o histórico do volume captado e não do volume consumido.

Dessa forma, o objetivo da pesquisa foi plenamente alcançado. Contudo, ainda existem questões a serem investigadas em relação a novos métodos de previsão e técnicas de combinação. O manuseio de séries temporais abrangendo outros fatores não apresentados nesse estudo, bem como a utilização da técnica de Redes Neurais Artificiais ou outras técnicas de combinações por exemplo, estabelece uma gama de possibilidades para realização de trabalhos futuros.

\section{AGRADECIMENTOS}

Agradecimentos à Fundação Araucária pela bolsa PIBIC concedida ao primeiro autor. Um agradecimento especial à Companhia de Saneamento do Sul do Brasil por fornecer os dados para o desenvolvimento dessa pesquisa. 


\section{REFERÊNCIAS}

ANA - Agência Nacional de Águas. ATLAS BRASIL: abastecimento urbano de água: panorama nacional. Engecorps/Cobrape - Brasília: ANA: Engecorps/Cobrape, 2010.

ANDRADE, J. S. Apontamentos de econometria aplicada. 2004. Disponível em: $<$ http://www4.fe.uc.pt/jasa/estudos/econometria.pdf $>$. Acesso em: set. 2015.

AREAL, N. M. de P. B. da C.; ARMADA, M. J. da R. Testes paramétricos e nãoparamétricos de reversão para a média da rendibilidade de índices do mercado accionista. Rev. adm. contemp. [online]. v.3, n.2, p. 7-28, 1999. Disponível em: <http://dx.doi.org/10.1590/S1415-65551999000200002>. doi: 10.1590/S141565551999000200002.

ARMSTRONG, J. S. Principles of Forecasting: A Handbook for Researchers and Practitioners. Kluwer Academic Publishers. 2001.

BERTRAND, J.; FRANSOO, J. Operations Management Research Methodologies Using Quantitative Modeling. Internacional Journal of Operations \& Production Management. v.22, p. 241-264, 2002. Disponível em: $<$ http://dx.doi.org/10.1108/01443570210414338>. doi: 10.1108/01443570210414338.

BOX, G.E.P.; JENKINS, G.M.; REINSEL, G.C.; LJUNG, G.M. Time series analysis: forecasting and control. 5 ed., New Jersey: Wiley. 2016.

CMMAD - Comissão Mundial sobre Meio Ambiente e Desenvolvimento. Nosso futuro comum. 2. ed. Rio de Janeiro: Editora da Fundação Getúlio Vargas. 1991.

FAVA, V. L. Manual de econometria. In: VASCONCELOS, M. A. S.; ALVES, D. São Paulo: Editora Atlas, 2000.

HYNDMAN, R. J.; ATHANASOPOULOS, G. Forecasting: principles and practice. 2016. Disponível em: <http://otexts.org/fpp/>.

HYNDMAN, R. J.; KHANDAKAR, Y. Automatic Time Series Forecasting: The forecast Package for R. Journal of Statistical Software. v.27, n.3, 2008. doi: 10.18637/jss.v027.i03.

IBGE. Instituto Brasileiro de Geográfica e Estatística. Estimativas populacionais para os municípios e para as Unidades da Federação brasileiros. 2016. Disponível em: $<$ http://www.ibge.gov.br/home/estatistica/populacao/estimativa2015/default.shtm>.

MAKRIDAKIS, S.; WHEELWRIGHT, S. C.; HYNDMAN, R. J. Forecasting: Methods and Applications. 3 ed., New York: John Wiley \& Sons, 1998.

MORETTIN, P. A.; TOLOI, C. M. de C. Análise de Séries temporais. São Paulo: Edgard Blucher, 2004. 
MUN, J. Modeling Risk: Applying Monte Carlo Risk Simulation, Strategic Real Options, Stochastic Forecasting, and Portfolio Optimization. 2. ed., New York: John Wiley \& Sons, 2010.

NEWBOLD, P.; BOS, T. Introductory Business \& Economic Forecasting. 2. ed., South-Western Publishing, Cincinnati. Ohio. 1994.

R DEVELOPMENT CORE TEAM. R: A language and environment for statistical computing. R Foundation for Statistical Computing, Vienna, Austria, 2011. Disponível em: <http://www.R-project.org/>.

R DOCUMENTATION. Box.test\{stats\} - Box-Pierce and Ljung-Box Tests. Package stats version 3.2.1, 2015.

RSTUDIO. 2015. Disponível em: <http://rstudio.org>.

WERNER, L. Um Modelo Composto para Realizar Previsão de Demanda através da Integração a Combinação e de Previsões e Ajuste Baseado na Opinião. Tese de Doutorado. Universidade Federal do Rio Grande do Sul, Porto Alegre, 2005.

WERNER, L.; RIBEIRO, J. L. D. Modelo composto para prever demanda através da integração de previsões. Produção, v.16, n.3, p. 493-509, 2006. Disponível em: $<$ http://dx.doi.org/10.1590/S0103-65132006000300011>. doi: 10.1590/S010365132006000300011.

WERNER, L.; RIBEIRO, J. L. D. Previsão de demanda: uma aplicação dos modelos Box-Jenkins na área de assistência técnica de computadores pessoais. Gestão \& Produção, v.10, n.1, p. 47-67, 2003. Disponível em: <http://dx.doi.org/10.1590/S0104-530X2003000100005>.

WPP. World Population Prospects. The 2015 Revision, Key Findings and Advance Tables Working Paper No. ESA/P/WP.241. United Nations, Department of Economic and Social Affairs, Population Division, 2015.

WUP. World Urbanization Prospects: The 2014 Revision, Highlights (ST/ESA/SER.A/352). United Nations, Department of Economic and Social Affairs, Population Division, 2014.

WWAP. World Water Assessment Programme. The United Nations World Water Development Report 4: Managing Water under Uncertainty and Risk. Paris, UNESCO, 2012. 